\title{
El género Geonoma (Arecaceae: Arecoideae) en México
}

\section{The genus Geonoma (Arecaceae: Arecoideae) in Mexico}

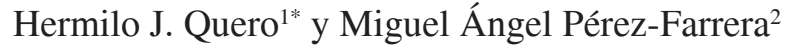 \\ ${ }^{1}$ Jardín Botánico, Instituto de Biología, Universidad Nacional Autónoma de México, Apartado postal 70-614, 04510 México, D.F., México. \\ ${ }^{2}$ Escuela de Biología, Universidad de Ciencias y Artes de Chiapas (UNICACH). Libramiento Norte Poniente s/n, Col. Lajas-Maciel, Tuxtla Gutiérrez, \\ Chiapas, México. \\ *Correspondencia: quero@servidor.unam.mx
}

\begin{abstract}
Resumen. Se hizo un estudio taxonómico de las palmas del género Geonoma Willdenow en México mediante recolecciones y observaciones morfológicas y ecológicas de esas palmas en diversas partes del país, y se revisaron ejemplares de herbarios nacionales y extranjeros. Las especies que se reconocen para México son: G. interrupta (Ruiz et Pavón) Mart., G. membranacea H. Wendl. ex Spruce y G. undata Klotzsch. Se proporciona una clave y descripciones de las especies, así como fotografìas para ilustrarlas.
\end{abstract}

Palabras clave: Geonoma, palmas, Arecaceae, México, Chiapas, Veracruz, Tabasco, Oaxaca.

\begin{abstract}
A review of Geonoma (Arecaceae: Arecoideae, Geonomateae) was made in order to know the number of species occurring in Mexico. The review included examination of specimens housed in Mexican and foreign herbaria; additional specimens were collected in southern Mexico, to record morphological and ecological observations in the field. The species recognized for Mexico are G. interrupta (Ruiz et Pavón) Mart., G. membranacea H. Wendl ex Spruce. and G. undata Klotzsch. A key to species and descriptions, and some photographs are provided.
\end{abstract}

Key words: Geonoma, palms, Arecaceae, Mexico, Chiapas, Veracruz, Tabasco, Oaxaca.

\section{Introducción}

Geonoma es un género de palmas (Arecaceae) perteneciente a la subfamilia Arecoideae, tribu Geonomateae, (Dransfield et al., 2005). La mayoría de sus especies son de origen sudamericano, aunque también se encuentran en Centroamérica y el Caribe, siendo México su límite más al norte.

El género fue descrito por Willdenow (1805), tipificándolo con la especie Geonoma simplicifrons; el número de especies reconocidas ha ido variando. Willdenow (1805) menciona alrededor de 180 especies, mientras que Wessels-Boer (1968) sólo reconoce algo más de 80; Uhl y Dransfield (1987) más de 75; Henderson et al. (1995) registra 51, y el mismo Henderson (2005), en un trabajo inconcluso dado a conocer en internet y siguiendo algunos criterios de Wessels-Boer (1968), sólo menciona 30 especies y 29 subespecies.

Las especies de este género son de lo más diversas, lo que dificulta su identificación; es muy frecuente encontrar ejemplares de la misma especie, creciendo en la misma

Recibido: 19 marzo 2008; aceptado: 25 enero 2010 población, con hojas divididas en un número variable de segmentos, así como el ancho de los mismos; en cuanto a las inflorescencias, su tamaño y ramificación varían dependiendo de la edad y altura de los ejemplares. Lo más constante es la forma de las hendiduras florales y su distribución en las raquillas.

\section{Materiales y métodos}

Se hizo una revisión bibliográfica de artículos y libros que mencionan nombres de especies de Geonoma o que describen nuevas especies para México o para otros países pero que podrían estar en México; se hizo hincapié en las revisiones más importantes e integrales del género, como las de Burret (1930), Wessels-Boer (1968) y la página web de Henderson (2005). Se revisaron los herbarios MEXU, XAL, HEM, CAS, US, NY, que por su ubicación geográfica o por sus programas de investigación tienen en sus colecciones ejemplares de Geonoma. Adicionalmente, se recolectó en Veracruz, Tabasco, Oaxaca y Chiapas, que son los estados donde se distribuyen las especies del género, y como complemento se hicieron observaciones ambientales, ecológicas y mediciones morfológicas. 
Geonoma. Willdenow, Species Plantarum 4(1):174, 1805. Lectotipo: G. simplicifrons Willdenow, Ing. Pl. 4, 594, 1805. Tipo: Venezuela. Caracas, sin fecha, F. Bredemeyer 20 (holotipo: B, imagen del holotipo Neg. del F. 16092). Kalbreyera Burret, Botanische Jahrbücher für Systematik 63: 142. 1930. Tipo: K. triandra Burret.

Taenianthera Burret, Botanische Jahrbücher für Systematik 63: 267. 1930. Tipo: T. macrostachys (Mart.) Burret.

Palmas monoicas, pequeñas o de mediano tamaño, inermes con tallos delgados. Hojas 6-20 por tallo, pinnadas o enteras; pinnas con divisiones irregulares, pocas o numerosas y anchas o delgadas lineares o sigmoides, pero siempre en el mismo plano; pecíolo corto o largo. Inflorescencias inter o infrafoliares, espigadas poco o muy ramificadas, con 2 espatas caducas antes de la antesis. Flores unisexuales pequeñas, dimorfas, glumiformes, embebidas en hendiduras del ráquis y pueden ser mono o bilabiadas, en grupos de 3 , la de en medio femenina, arregladas en espiral, rara vez en verticilos; flores masculinas con sépalos imbricados, pétalos unidos en la base y valvados arriba; estambres generalmente 6, filamentos fusionados en la base formando un tubo, anteras bífidas, pistilodio generalmente presente; flores femeninas con sépalos imbricados en botón, libres o ligeramente fusionados en la madurez, pétalos fusionados abajo, valvados arriba, estaminodios formando un tubo coroliforme rodeando al pistilo. Fruto pequeño, globoso u ovoide, remanente estigmático basal, con epicarpo liso, delgado, mesocarpo delgado, carnoso y fibroso, endocarpo cartáceo. Semilla 1, libre, globosa o subglobosa; endospermo homogéneo; embrión basal.

Las especies del género, son características de regiones neotropicales; crecen a la sombra de otras plantas en altitudes cercanas al nivel del mar y hasta alrededor de los $2500 \mathrm{~m} \mathrm{snm}$.

\section{Comentarios taxonómicos}

Existen algo más de 50 especies en las regiones tropicales, desde el sur de México hasta Brasil y Bolivia. Para México se habían mencionado 8 especies: posteriormente, sólo se mencionó $G$. oxycarpa, que pasó a sinonimia de G. interrupta (Henderson et al., 1995).

Con base en recolecciones y revisiones de herbarios se concluye que en México hay 3 especies: $G$. interrupta, $G$. membranacea y G. undata, en regiones cálido-húmedas de los estados de Veracruz, Oaxaca, Tabasco y Chiapas.

Geonoma interrupta (Ruiz et Pavón) Mart., Hist. Nat. Palm. 2: 8, t 7. 1823. Basónimo: Martinezia interrupta Ruiz et Pavón, Syst. Veg. 296. 1798. Perú, entre Pozuzo y Cuchero. Ruiz y Pavón s.n. (F, K y M). Fig. 1.

Geonoma mexicana Liebm. ex Mart., Hist. Nat. Palm. 3: 316. 1850. Tipo: México, Oaxaca, entre Chinantla y Teovela, Liebmann 10804 (holotipo C; fototipo F).

Geonoma binervia Oerst., Vidensk. Medel. Dansk. Naturhist. Foren. Kjoebenhavn 33. 1858. Tipo Nicaragua, San Juan, Oersted 6554 (holotipo C).

Geonoma magnifica Linden et H. Wendl. Linnaea 28: 335. 1856. Tipo: México. Tabasco Ghiesbreght sn.

Palmas pequeñas con tallos solitarios, de 2-4 m de alto, 4$7 \mathrm{~cm}$ de diámetro. Hojas generalmente dividida en 3 anchos pares de segmentos, fuertemente nervados, $60-100 \mathrm{~cm}$ de largo; pecíolo 20-40 cm de largo; segmentos apicales 15-30 cm de largo, 10-20 cm de ancho, los segmentos medios 30$45 \mathrm{~cm}$ de largo, 14-18 cm de ancho, los segmentos inferiores 25-35 cm de largo, 8-15 cm de ancho. Inflorescencia hasta $70 \mathrm{~cm}$ de largo, 2-3 veces ramificada; pedúnculo 20-40 cm de largo; la profila y la bráctea peduncular, semejantes en tamaño, $15-30 \mathrm{~cm}$ de largo y hasta $5 \mathrm{~cm}$ de ancho, ambas fuertemente lepidotas; raquis $10-25 \mathrm{~cm}$ de largo; raquillas 10-30, gruesas, pubescentes, 7-16 cm de largo, hendiduras florales unilabiadas, con el labio entero, redondeado, no excediendo la yema floral; flores masculinas excediendo las hendiduras; flores femeninas con el tubo del estaminodio truncado, sobrepasando los pétalos; fruto globoso a ligeramente elipsoidal de 4-6 mm de diámetro,

\section{Clave para las especies de Geonoma en México}

Palmas de mediano tamaño, de 4-10 m de alto y hasta $10 \mathrm{~cm}$ de diámetro, raquillas de 3-7 mm de diámetro; hendiduras florales bilabiadas; fruto ovoide de 8-10 $\mathrm{mm}$ de largo y 6-7 $\mathrm{mm}$ de diámetro. G. undata

Palmas pequeñas, menores de $4 \mathrm{~m}$ de alto; hendiduras florales unilabiadas, solo con el labio inferior; fruto globoso a muy ligeramente ovoide, menores de $8 \mathrm{~mm}$ de diámetro.

Inflorescencia hasta $70 \mathrm{~cm}$ de largo con numerosas ramas primaria, divididas de segundo y tercer orden; raquillas largas, fruto ovoide a ligeramente ovoide, apiculado en el ápice G. interrupta Inflorescencia pequeña de menos de $45 \mathrm{~cm}$ de largo, escasamente ramificada, ramas inferiores bífidas y superiores simples, fruto ovoide no apiculado en el ápice 


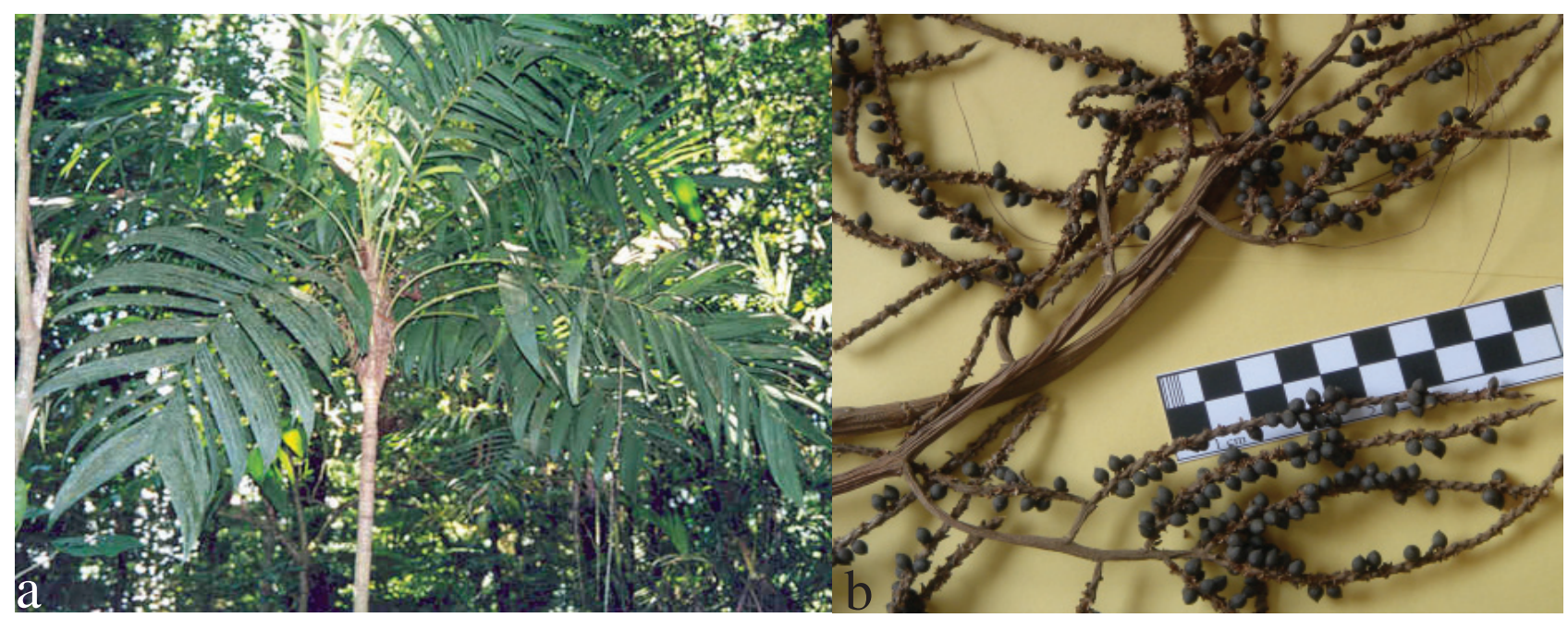

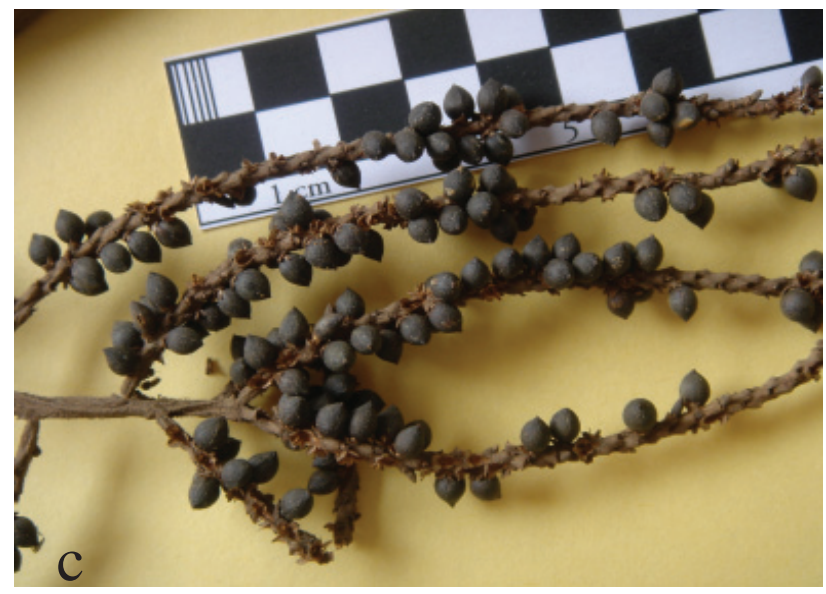

Figura 1. Geonoma interrupta: a), ejemplar en su medio natural; b-c), ejemplares de herbario en donde se pueden observa frutos maduros, pequeños y apiculados.

negro a púrpura en la madurez; semilla globosa, 3-4 mm de diámetro.

Ejemplaresexaminados. CHIAPAS:MunicipiodeJaltenango, Reserva de la Biosfera El Triunfo, sendero Finca PrusiaCampamento El Triunfo, N. Martínez Meléndez 596 (HEM); Municipio de Mapastepec, Sierra Madre de Chiapas, $5 \mathrm{~km}$ al N del ejido Loma Bonita, M. A. Pérez Farrera 1283 (HEM, MEXU); Municipio de Marqués de Comillas: cerro camino a Tzendales, 2 jornadas del Cedro, F. Miranda 8471; Estación Biológica de Chajul, Vásquez 258 (CAS); Municipio de Ocosingo, Crucero Bethel, G. Aguilar 3920 (MEXU); ruins of Yaaxchilan, Río Usumacinta, D. Breedlove 33939 (CAS); ruins of Yaaxchilan, Río Usumacinta, D. Breedlove 34178 y McKlinton (CAS); centro arqueológico de Bonampak, $J$. Meave et al. B 251 (MEXU); Municipio de Ocozocoautla de
Espinosa, $46 \mathrm{~km}$ al N of Ocozocoautla, D. Breedlove 38686 (CAS); Municipio de Tapachula: Finca Mexiquito, Purpus 7623 (CAS); Municipio de Tecpatán, sureste de cerro Mono Pelón, S. Zamudio 1507 (MEXU); ejido San Antonio, $3 \mathrm{~km}$ al oeste del lago Pujol, H. Quero 3614 (MEXU). OAXACA: Municipio de Santa María Chimalapa; 15 km al ESE de Santa María, H. Hernández 1434 (MEXU); Municipio de Tuxtepec, $8 \mathrm{~km}$ al oeste de Valle Nacional, H. Quero 3007 (MEXU); $1 \mathrm{~km}$ al S de Breña Torres, $H$. Quero 3479 (MEXU). TABASCO: Municipio de Cárdenas, Km. 25 carretera Cárdenas-Coatzacoalcos, M. Magaña 1605 y S. Zamudio (MEXU). Municipio de Huimanguillo, El Bernal, H. Puig 701 (MEXU); VERACRUZ. Municipio de Atoyac: La Junta, C. B. Doyle \& G. N. Collins 10 (US); Municipio de Catemaco, camino a Bastonal, A. Vovides 566 (XAL); barranca de Sontecomapan, L. Nevling y A. GómezPompa 160, 181 (XAL); Municipio de Coatzacoalcos, Buena Aventura, near Coatzacoalcos, C. B. Doyle \& G. N. Collins 6 (US); Municipio de Emiliano Zapata: Dos Rios, C. D. Mell 653 (US); Municipio de Hidalgotitlán, ejido Agustín Melgar, $4 \mathrm{~km}$ al SE del campamento Hermanos Cedillo, I. Calzada 10470, 10483 (XAL); reserva ejidal, junto al Cerro Amarillo, G. Castillo 333 (XAL); $2 \mathrm{~km}$ al E. del campamento Hermanos Cedillo, camino a la Laguna, $J$. Dorantes 2499 (XAL); Municipio de San Andrés Tuxtla, estación de Biología Los Tuxtlas, I. Calzada 813 (MEXU); Estación de Biología Los Tuxtlas, A. Gómez-Pompa 4466 (MEXU); Estación de Biología Los Tuxtlas, G. Ibarra et al. 2021, 21443137 (MEXU, XAL); Estación de Biología Los Tuxtlas, H. Quero 3134, 3547 (MEXU).

Esta especie es la más común en México, se le encuentra en selvas altas perennifolias de los estados de Veracruz, Oaxaca, Chiapas y Tabasco, en altitudes de 500 a 1500 m snm. 


\section{Comentarios taxonómicos}

Es de las especies con mayor variabilidad morfológica; por mucho tiempo se le identificó como G. oxycarpa, pero Henderson (1995) estima que esta especie junto con otras deberían pasar a sinonimia; sin embargo, el mismo Henderson (com. pers.) considera que $G$. interrupta es un complejo de especies, del cual $G$. oxycarpa podría ser excluida a futuro. Standley (1926) menciona para México $G$. mexicana y $G$. magnifica, y si bien para Wessel-Boer son coespecíficas de G. oxycarpa, Henderson (2005) las considera subespecies de G. interrupta. Por otra parte, G. preussii fue descrita para México por Wessels-Boer (1968) como coespecífica de $G$. interrupta. De acuerdo con este autor, la consideramos coespecífica de G. interrupta.

Geonoma membranacea H. Wendl. ex Spruce, J. Linn. Soc. 11: 106. 1869. H. Wendl. s. n., Guatemala, volcán del Fuego (holotipo: K). Fig. 2.

Geonoma leptoclada Burret, Notizbl. 11: 863. 1933: Lehmann 1613 . Guatemala: volcán de Santa María (tipo destruido en B).

Palmas pequeñas, con tallos solitarios de 2-4 m de alto y 2-4 cm de diámetro, entrenudos de $3-5 \mathrm{~cm}$ de largo, hojas alrededor de $1.5 \mathrm{~m}$ de largo, vaina de $10-15 \mathrm{~cm}$ de largo, pecíolos de $20-30 \mathrm{~cm}$ de largo, lámina de $60-80 \mathrm{~cm}$, dividida en 5-11 pares de segmentos desiguales de 20-40 cm de largo, los apicales de 7-11 cm de ancho con 10-12 nervaduras prominentes. Inflorescencia pequeña, interfoliar, de 30-45 $\mathrm{cm}$ de largo, 2 veces ramificada, con 5-8 ramas primarias, con 2 brácteas tubulares, cartáceas, pedúnculo de $10-20 \mathrm{~cm}$ de largo, ramas primarias bífidas abajo, indivisas arriba, raquillas de 10 a $25 \mathrm{~cm}$ de largo y 1.5-2.0 mm de diámetro, hendiduras florales sólo con labio inferior, truncado o redondeado, arregladas en una espiral muy laxa; frutos globosos a subglobosos de 5-7 $\mathrm{mm}$ de diámetro, semillas globosas de 4-6 mm de diámetro.

Ejemplares examinados. CHIAPAS: Municipio de Jaltenango: Cacatale, Escuintla, E. Matuda 16384 (MEXU); La Gloria, El Triunfo, Escuintla, E. Matuda 18568 (MEXU); camino Santa Rita al campamento de El Triunfo, H. Quero 3888 y 3889 y M. A. Pérez Farrera (HEM, MEXU) Barranca de Aguascalientes, F. Miranda 1727 (MEXU). Municipio de Unión Juárez: Monte Ovando, E. Matuda 3963 (CAS, MEXU).

Esta especie sólo se había encontrado en Guatemala y había colectas de Chiapas identificadas erróneamente como G. oxycarpa y G. interrupta. Después de recolectar ejemplares en esa región, se confirmó la existencia de G. membranacea en México, en selvas altas y medianas perennifolias.

\section{Comentarios taxonómicos}

Geonoma membranacea también fue considerada por Henderson (1995) como coespecífica de G. interrupta; el mismo Henderson, en su trabajo inédito, la coloca como una subespecie de G. pinnatifrons; sin embargo, hay diferencias en la inflorescencia y en el fruto como para mantenerla como una especie válida; tiene mucha similitud con G. leptoclada, por lo que estamos de acuerdo con Wessels- Boer (1968) de que es coespecífica de G. membranacea.

Geonoma undata Klotzsch, Linnaea 20: 452. 1847. H Karsten 26, Venezuela, sin datos (holotipo: LE; isotipo: BM). Fig. 3.

Geonoma seleri Burret, Bot. Jahrb. 63: 211. 1930. E. Seler 2757, Guatemala: Huehuetenango, Yalambohoch (destruido en B).

Geonoma polyneura Burret, Notizbl. 11: 500. 1932. Cook \& Griggs 36, Guatemala: Alta Vera Paz, Sepacuite (holotipo US!).

Palmas con tallos solitarios, hasta $9 \mathrm{~m}$ de alto y de 6-10 cm de diámetro; hojas hasta $2.5 \mathrm{~m}$ de largo, vaina de 30-60 cm de largo, café lepidota, pecíolo 40-50 cm de largo lamina de 1.40-1.60 cm de largo, dividida en 12-30 segmentos por lado, anchos o angostos, regular o irregularmente distribuidos, los apicales de 30-40 cm de largo y 10-13 cm de ancho, los medios de 35-40 cm de largo y 2-7 cm de ancho, los basales $30-40 \mathrm{~cm}$ de largo y $3-7 \mathrm{~cm}$ de ancho con nervaduras primarias prominentes en ambas superficies, las secundarias mas prominentes en el envés, las terciarias conspicuas en ambas superficies. Inflorescencia infrafoliar, con ramificaciones de $2^{\circ}$ y 3 er orden, pedúnculo con pubescencia café tomentulosa, de $30-50 \mathrm{~cm}$ de largo, raquillas rojizas en la madurez, de 10-40 cm de largo y 4-6 mm de grosor; hendiduras florales bilabiadas, la superior emarginada, la inferior bifurcada, Frutos ovoides, puntiagudos de 8-10 mm de largo y 6-8 mm de diámetro; negros en la madurez.

Ejemplares examinados. CHIAPAS: Municipio de la Concordia: Reserva de la Biosfera El Triunfo, Polígono IV, cerro Soconusco, $5 \mathrm{~km}$ al sur de Rancho San Martín de Porres, $R$ Martínez Camilo 124 (HEM); Municipio de Jaltenango, camino del Triunfo a Finca Prusia, Calzada, I. et al. 8966 (XAL); Reserva El Triunfo, polígono 1, M. Heath y A. Long 481, 985 (CAS, MEXU); Finca Prusia, arroyo Charco, $F$. Miranda 6971 (MEXU); Reserva de la Biosfera El Triunfo, Campamento Triunfo-Palo Gordo, M. A. Pérez Farrera 1122 (HEM, MEXU); camino al Campamento del Triunfo, $H$. Quero 3884 y M. Pérez Farrera (HEM, MEXU). Municipio Villa de Corso; Base of Cerro Tres Picos, near Cerro Bola, SW of Colonia Agrónomos Mexicanos, D. Breedlove 24107 

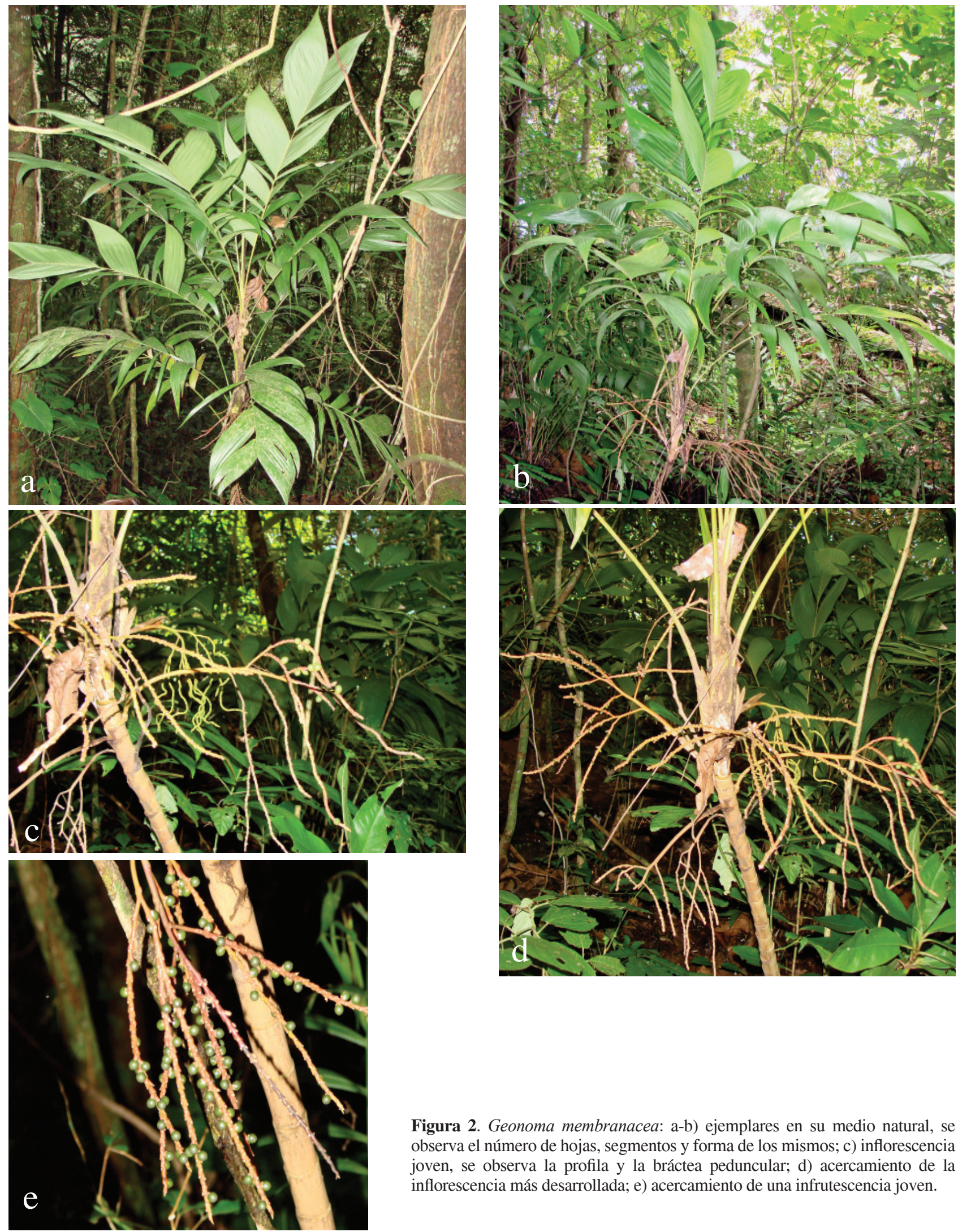

Figura 2. Geonoma membranacea: a-b) ejemplares en su medio natural, se observa el número de hojas, segmentos y forma de los mismos; c) inflorescencia joven, se observa la profila y la bráctea peduncular; d) acercamiento de la inflorescencia más desarrollada; e) acercamiento de una infrutescencia joven. 

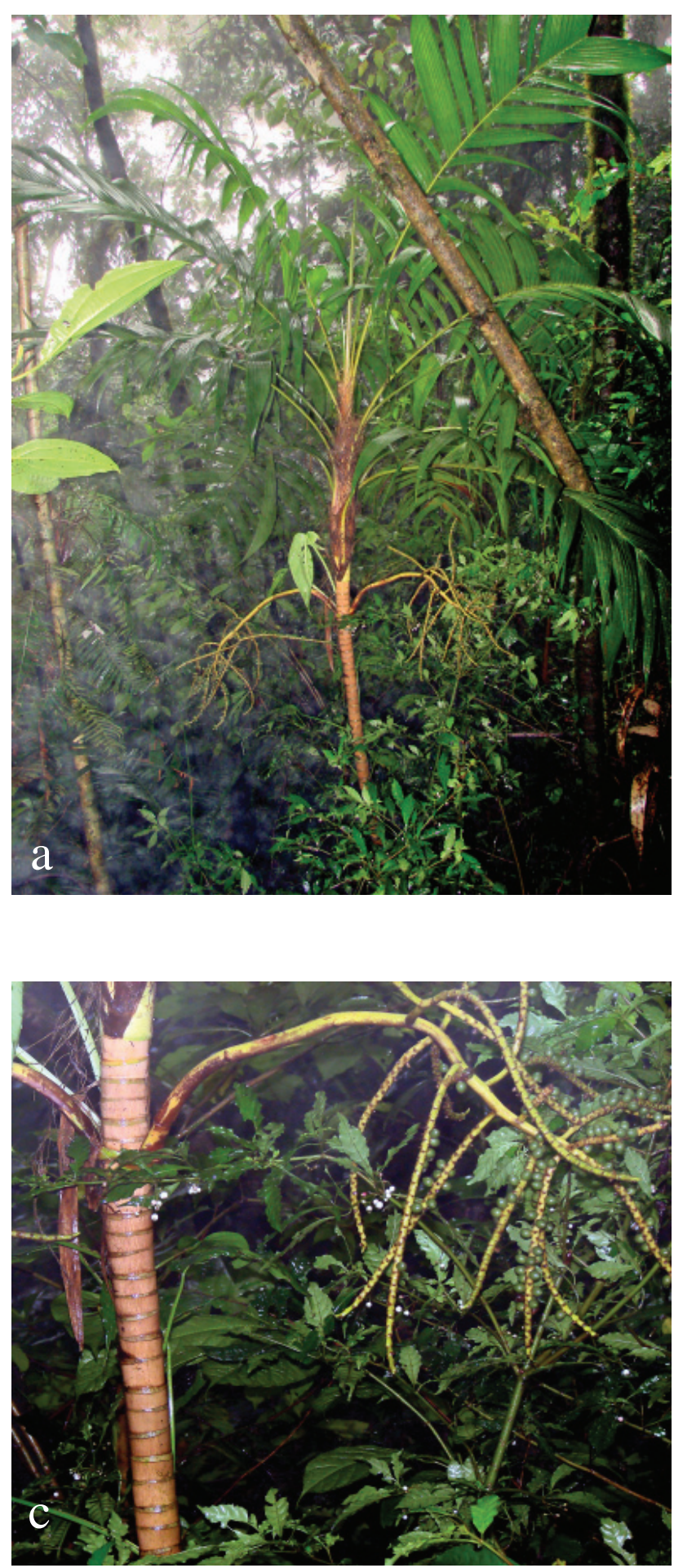

Figura 3. Geonoma undata: a-b) ejemplar en su medio natural, mostrando número de hojas, segmentos y forma de los mismos; c) acercamiento donde se observa la inflorescencia infrafoliar y los entrenudos del tallo; d) acercamiento de la inflorescencia, donde se puede observar la bráctea peduncular muy larga
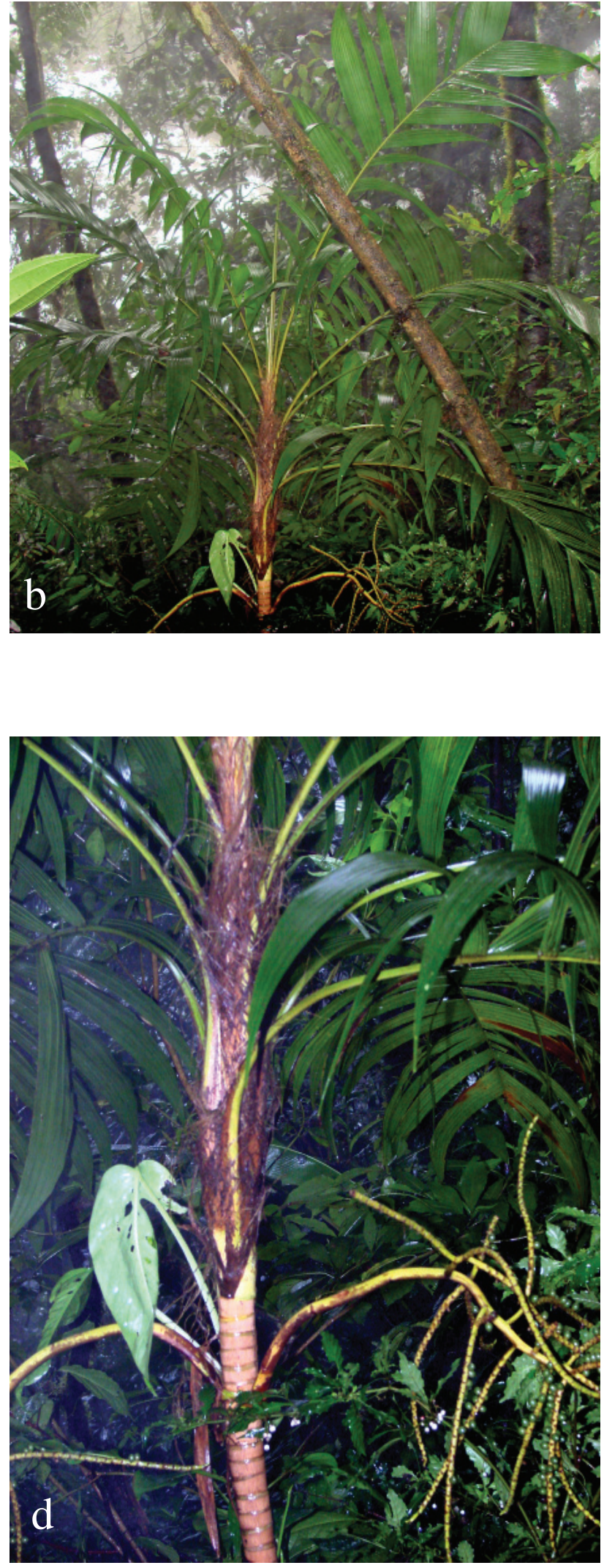
(CAS); SE side of Cerro Tres Picos, D. Breedlove 34415 (CAS); Municipio Villa Flores: ejido Nueva Independencia, cerro Tres Picos, M. Pérez Farrera s/n (MEXU); cerro Las Minas, cerca de la Finca Santa Cruz, M. A. Pérez Farrera 1825 (HEM); Municipio de la Trinitaria, Laguna Tzikaw, Monte Bello, D. Breedlove 35114 (CAS, MEXU); $20 \mathrm{~km}$ de Colonia Cuauhtemoc; $A$. Méndez 7848 (MEXU); Laguna de Montebello, F. Miranda 2694 (MEXU); TABASCO: Municipio de Huimanguillo: Villa de Guadalupe a Torre microondas Huimanguillo, A. Guadarrama y G. Ortiz 4122 (MEXU).

\section{Comentarios taxonómicos}

Esta especie, con el nombre de G. seleri, fue reconocida por mucho tiempo como endémica de Guatemala y luego encontrada en México, posteriormente fue considerada por Henderson (1995) como coespecífica de G. undata, situación con la cual coincidimos a pesar de que en su trabajo posterior en internet, este autor excluye la especie $G$. seleri y establece la subespecie $G$. undata subsp. edulis para las poblaciones de México. Por tanto, la distribución de G. undata se extiende desde el sur de México hasta Perú y Bolivia. S e le encuentra en selvas perennifolias y bosques mesófilos, de la sierra Madre de Chiapas entre los 1800 y los 2200 m snm.

\section{Discusión}

En este trabajo se ha determinado que en México existen 3 especies del género Geonoma: G. interrupta, G. membranacea y G. undata. En nuestro país se habían descrito G. preussi, G. mexicana y G. magnifica, las cuales, coincidimos en que son coespecíficas de $G$. interrupta. También se sugirió que podrían estar presentes $G$. membranacea y $G$. leptoclada, descritas de Guatemala (Steyermak, 1958). Al revisar material de ambas especies no encontramos diferencias entre ellas, por lo que consideramos que G. membranacea es la especie correcta. Geonoma seleri fue descrita de Guatemala, en las cercanías con México, y registrada como endémica de ese país, aunque posteriormente se colectó en Chiapas, México. La especie fue considerada correcta por Wessels-Boer (1968) y R. W. Read (1979); sin embargo, al revisar ejemplares de US y NY observamos que no existen diferencias significativas entre $G$. seleri y $G$. undata, por lo que concordamos con Henderson (2005) en que la especie correcta es $G$. undata.

En cuanto a $G$. interrupta, está claro que es una de las especies del género con mayor variabilidad morfológica, tanto en el tamaño de las hojas y en la división de éstas, como en la ramificación de las inflorescencias y las características de las hendiduras florales, por lo que es verosimil que a futuro sufrirá cambios nomenclaturales, como lo ha estado intentando Henderson (2005) y que en forma provisional lo ha llevado a dividir esta especie en varias subespecies; sin embargo, dicho autor considera que terminar su monografía le llevará varios años. Por lo que independientemente de lo que Henderson está trabajando, se considera que $G$. interrupta, G. membranacea y G. undata. son las 3 especies que crecen en México.

\section{Agradecimientos}

A los biólogos Nayeli Martínez y Rubén Martínez, de la Escuela de Biología de la UNICACH, por su valiosa ayuda en los trabajos de campo y en el procesamiento de las muestras. Al Fondo Sectorial CONACYT-SEMARNAT, proyecto clave SEMARNAT-2004-C01-272, por el apoyo en algunas salidas de campo.

\section{Literatura citada}

Bailey, L. H. 1939. Geonomes. In The Lesser Antilles. Gentes Herbarium. Bailey Hortorium, Cornell University, Ithaca, New York 4:226-227.

Burret, M. 1930. Geonomae Americanae. Botanische Jahrbucher fur Systematik pflansengeschichte und pflanzengeographie. Leipzig. 63:123-270.

Dransfield, J., N. Uhl, C. B. Asmussen, W. J. Baker, M. M. Harley y C. E. Lewis. 2005. A new phylogenetic classification of the palm family, Arecaceae. Kew Bulletin 60:559-569.

Henderson, A., G. Galeano y R. Bernal. 1995. Field guide to the palms of the Americas. Princeton University Press, New Jersey. 352 p.

Henderson, A. 2005. Geonoma. Internet, New York Botanical Garden: http://www.Nybg.Org/Botany/Geonoma/Index.Html; last access: 21.X.2006.

Moore, H. E. 1968. The geonomoid palms. Taxon 18:230-232

Read, R. W. 1979. Palmae. In Flora of The Lesser Antilles, Arnold Arboretum. Harvard University 3:364-368.

Standley, P. C. 1920. Trees and shrubs of Mexico. Contribution to The National Herbarium, Smithsonian Institution of Washington 23:76.

Steyermark, J. 1958. Palmae. In Flora de Guatemala, P. C. Standley y J. Steyermark.. Fieldiana Botany 24:266-271.

Uhl, N. W. y J. Dransfield, 1987. Genera palmarum. L. H Bailey Hortorium and The International Palm Society. Allen Lawrence, Kansas. p. 539-541.

Wessels-Boer, J. G. 1968. The geonomoid palms. Verhandelingen der Konninklijke Nederlandse Akademie van Wetenschappen, Afdeling Natuurkunde. Tweede Sectie 58:202 p. 